\title{
Nonanalytic Vapor Pressure Equation With Data for Nitrogen and Oxygen
}

\author{
Robert D. Goodwin* \\ Institute for Basic Standards, National Bureau of Standards, Boulder, Colorado 80302
}

(June 10, 1969)

\begin{abstract}
The specific heat of a two phase liquid-vapor system at constant volume apparently increases without limit at temperatures approaching the critical point, suggesting (via a thermodynamic relation) that the vapor pressure derivative $d^{2} P / d T^{2}$ may behave similarly. This nonanalytic behavior at the critical point is used in the present vapor pressure formula to gain simplicity and accuracy, as seen by use of data for nitrogen and oxygen.
\end{abstract}

Key words: Critical point; equation; formula; liquid; nitrogen; nonanalytic; oxygen; vapor pressure.

\section{List of Symbols}

$A, B, C \quad$ constant coefficients. $a, b, c, d, e$ constant coefficients. $\Delta \quad 100 \cdot\left(P / P_{\text {calc }}-1\right)$. $\epsilon \quad$ a nonintegral exponent, $1<\epsilon<2$. $F(x) \quad$ any function of $T$, nonanalytic at $T_{c}$. $P \quad$ pressure, 1 atm $=0.101325 \mathrm{MN} / \mathrm{m}^{2}$. $P_{c} \quad$ critical-point pressure. $P_{t} \quad$ triple-point pressure. $T \quad$ temperature, $\mathrm{K}$. $T_{c} \quad$ critical-point temperature. $T_{t} \quad$ triple-point temperature.

$x(T) \equiv\left(1-T_{t} / T\right) /\left(1-T_{t} / T_{c}\right)$. $y(P) \equiv \log \left(P / P_{t}\right) / \log \left(P_{c} / P_{t}\right)$.

\section{Introduction}

An accurate vapor pressure equation is essential for computing thermodynamic properties of fluids, but a satisfactory function remains undiscovered [1]. ${ }^{1}$ To achieve accuracy for thermal property computations, many authors resort to polynomial representations of experimental vapor pressure data. This has the serious disadvantage that derivatives, $d P / d T$ etc., may not be reliable.

A new approach to this problem resides in the proposal that $d^{2} P / d T^{2}$ be infinite at the critical point, as given by a term $P \sim-\left(T_{c}-T\right)^{2} \cdot \log \left(T_{c}-T\right)$ where subscript $c$ refers to the critical point [2]. Other work on critical phenomena [3] suggests that this pole also might be described by use of a nonintegral exponent $\epsilon$ in a term $\left(T_{c}-T\right)^{\epsilon}, 1<\epsilon<2$.

*Cryogenics Division, NBS Boulder Laboratories, Boulder, Colorado, 80302.

1 Tigures in brackets indicate the literature references at the end of this paper.
Our particular need was for a reduced equation sufficiently simple to permit examination of existing data on fluorine. I have therefore used data only on the similar substances nitrogen and oxygen in the present work. Application of this equation to new data on fluorine will be reported independently [4].

\section{Data and Method}

I have examined nitrogen and oxygen for deviations from the basic vapor pressure equation,

$$
\log (P)=a-b / T,
$$

as have many previous workers. To normalize the variables, I eliminate the constants of (1) by use of triple-point and critical-point properties in the definitions.

$$
\begin{aligned}
& x(T) \equiv\left(1-T_{t} / T\right) /\left(1-T_{t} / T_{c}\right), \\
& y(P) \equiv \log \left(P / P_{t}\right) / \log \left(P_{c} / P_{t}\right),
\end{aligned}
$$

where subscript $t$ refers to the triple point. These variables range from zero to unity. Equation (1) now reads simply $y=x$, and we may conveniently examine plots of the deviations $(y-x)$ as a function of $x$. For each substance the plot is qualitatively a simple cubic, with roots $x_{1}=0,0<x_{2}<1, x_{3}=1$.

\section{The Vapor Pressure Equation}

Qualitative behavior of the above deviations is

$$
(y-x)=A \cdot x \cdot(B-x) \cdot(1-x) .
$$


Precise oxygen vapor pressures [5] still cannot be represented accurately by (2). Addition of a nonanalytic term, $F(x)$, however, yields an accurate equation for nitrogen and oxygen,

$$
y=x+A \cdot x \cdot(B-x) \cdot(1-x)+C \cdot F(x) .
$$

Equation (3) is converted to a working equation by multiplying the terms in brackets and collecting powers of $x$,

$$
\log \left(P / P_{t}\right)=a \cdot x+b \cdot x^{2}+c \cdot x^{3}+d \cdot F(x) .
$$

This equation is used throughout the following work. It is constrained to a given triple-point pressure, $P_{t}$. As vapor pressures in the $\mathrm{mm} \mathrm{Hg}$ range have very low relative accuracy, figure $1 \mathrm{a}$, this constraint is desirable and useful. If $P_{t}$ is unknown and is to be estimated from the equation, we have the following option with no constraints,

$$
\log (P)=a+b \cdot x+c \cdot x^{2}+d \cdot x^{3}+e \cdot F(x) .
$$

Excellent representation of vapor pressure data for nitrogen and oxygen is obtained with either of the nonanalytic forms

$$
\begin{aligned}
& F_{1}(x)=-(1-x)^{2} \cdot \log (1-x), \\
& F_{2}(x)=x \cdot(1-x)^{\epsilon}
\end{aligned}
$$

where exponent $\epsilon$ is nonintegral, $1<\epsilon<2$. In the following we shall see that $(4 \mathrm{~b})$ is better for oxygen as $T$ approaches $T_{c}$. For nitrogen, data of the precision necessary to choose between $(4 \mathrm{a})$ and $(4 \mathrm{~b})$ are not available, and hence I do not plot the nitrogen deviations in this report. Nitrogen results are given only to indicate that (3) is not unique for oxygen.

As a guide for comparing results given below, the most accurate vapor pressure equation currently available for oxygen probably is that of Hust and Stewart [9],

$$
\log (P)=\sum_{i=0}^{7} A_{i} \cdot T^{i}
$$

with eight coefficients. We note absence of a term $1 / T$. This is no oversight, as these authors intensively investigated many well-known forms [1].

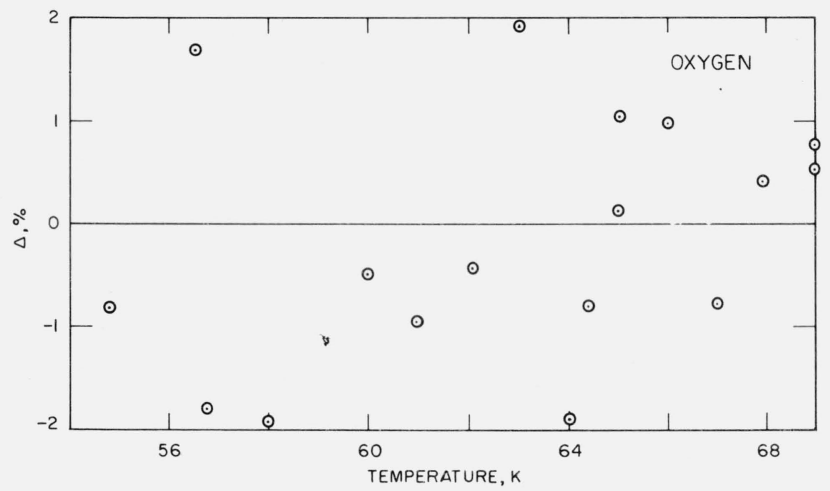

FigURE la. Low temperature deviations for oxygen with (3a) and $\mathrm{F}_{1}(\mathbf{x})$.

\section{Some Results for Nitrogen and Oxygen}

The nitrogen data used here are those selected by Strobridge [6], namely from [7] and [8]. The oxygen data are the same as selected by Hust [9], namely from [5]. Fixed-point constants used for the present work are in table 1. Results for eq (3a) are in table 2. Each column gives the form of $F(x)$, the derived criticalpoint pressure $P_{c}$, the relative critical-point slope, $[d \ln (P) / d \ln (T)]_{c}$, exponent $\epsilon$ and the coefficients, the number of datum pairs, $N P$, and the root mean square of individual, relative deviations, RMS.

Hoge [5] gives about 218 data for oxygen. For table 2 , some imprecise low-temperature data were eliminated to yield an rms deviation of 0.06 percent. For all 218 data the rms relative deviation is 0.34 percent, about the same as found by Hust and Stewart only by use of the eight-constant power series and by weighting the data and deviations according to their uncertainties. Our deviation was obtained with no weighting. and with fewer arbitrary coefficients.

Approaching the critical point, these oxygen data are extremely precise, enabling us to see that $F_{2}(x)$ gives a much better representation at $T>141 \mathrm{~K}$ than

\begin{tabular}{|c|c|c|}
\hline & Nitrogen & Oxygen \\
\hline 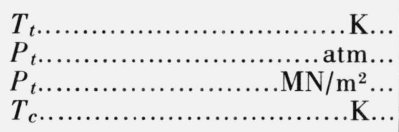 & 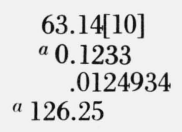 & $\begin{array}{l}54.353[5] \\
0.00150[5] \\
.0001519875 \\
154.77[5]\end{array}$ \\
\hline
\end{tabular}
does the logarithmic form, $F_{1}(x)$. This is evident upon comparing deviations via $F_{1}(x)$ in figure 1 with the corresponding deviations via $F_{2}(x)$ in figure 2 . On

\begin{tabular}{|c|c|c|c|}
\hline & Nitrogen & Oxygen & Oxygen \\
\hline$F(x)$ & $(a)$ & $(a)$ & $(b)$ \\
\hline (........... & 33.524 & 50.105 & 50.139 \\
\hline$P_{c} \ldots \ldots \ldots \ldots \mathrm{MN} / \mathrm{m}^{2} \ldots$ & 3.396819 & 5.076889 & 5.080334 \\
\hline$(d \ln (P) / d \ln (T))_{c}$ & 5.8775 & 5.9203 & 6.0117 \\
\hline 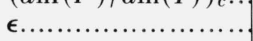 & & & 1.633 \\
\hline$a \ldots \ldots \ldots \ldots$ & 5.2149143 & 7.5955889 & 5.9479153 \\
\hline$b \ldots$ & 0.5126235 & 5.1202773 & 8.2487892 \\
\hline$c \ldots \ldots$ & -.1221435 & -2.2994456 & -3.7796060 \\
\hline d.... & .6158760 & 3.2246189 & 4.8725595 \\
\hline$N P$ & 89 & 188 & 188 \\
\hline RMS.................... & 0.051 & 0.060 & 0.059 \\
\hline
\end{tabular}
these plots $\Delta \equiv 100 \cdot\left(P / P_{\text {calc }}-1\right)$.

TABLE 1. Fixed-point constants used

${ }^{a}$ Adjusted to minimize deviations.

TABLE 2. Some results for equation (3a)

Table 3 compares results from (5) with results from (3a) using the nonanalytic form (4b). The last two columns give percent deviations of first and second derivatives via (5) from values obtained via (3a). These are plotted in figure 3. It is interesting to see that the 

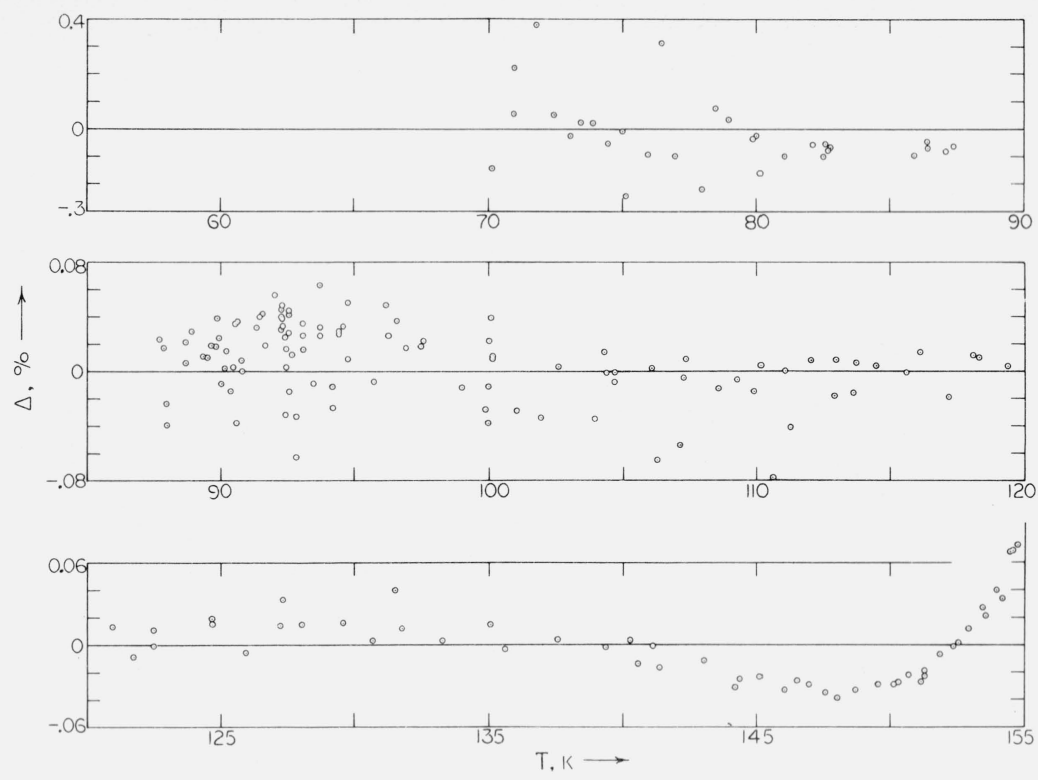

FIGURE 1. Deviations for oxygen with (3a) and $\mathrm{F}_{1}(\mathbf{x})$.
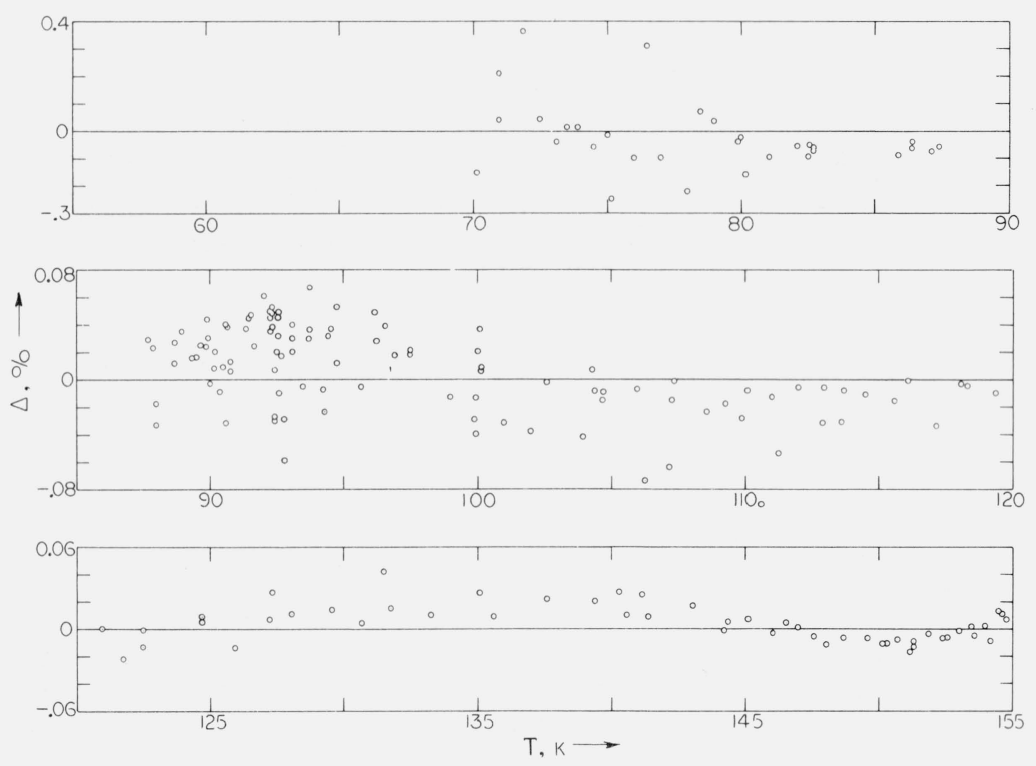

Figure 2. Deviations for oxygen with (3a) and $\mathrm{F}_{2}(\mathrm{x})$.

number of inversions on these plots corresponds exactly to the number of terms used in the power series (5).

We also may compare the curvature $d^{2} P / d T^{2}$ calculated by (3a) with results derived from our specific heat measurements on the two-phase, liquid vapor system for oxygen [11]. In the thermodynamic relation [2],

$$
\bar{C}_{n} / T=-d^{2} G / d T^{2}+\left(d^{2} P / d T^{2}\right) \cdot v,
$$

$\bar{C}_{v}$ is heat capacity of the two phase system at constant volume, $G$ is the Gibbs free energy per mol, and $v$ is the overall, average molal volume. Data for $C_{n},(T)$ at two or more different densities must be interpolated onto isotherms. Equation (6) then gives $d^{2} P / d T^{2}$ as the slope of plots of $\bar{C}_{v} / T$ versus $v$. Results have relatively large uncertainties, exceeding those expected from the vapor pressure equation. Table 4 gives this comparison of $d^{2} P / d T^{2}$ from (3a) with results from specific heat data via (6).

As a preliminary measure of the generality of (3), table 5 gives the constants obtained when using $F_{1}(x)$. These values were derived from results such as those in table 2. Fluorine results are quite preliminary. Constants for all three substances have the same signs and about the same magnitudes. 
TABLE 3. Comparison of oxygen vapor pressure equations

\begin{tabular}{|c|c|c|c|c|c|c|c|c|}
\hline \multicolumn{4}{|c|}{ Goodwin } & \multicolumn{3}{|c|}{ Hust-Stewart } & \multirow{2}{*}{$\begin{array}{l}\text { Percent } \\
d P / d T\end{array}$} & \multirow{2}{*}{$\begin{array}{l}\text { Percent } \\
d^{2} P / d T^{2}\end{array}$} \\
\hline$T, K$ & $P, A T M$ & $d P / d T$ & $d^{2} P \Gamma d T^{2}$ & $P, A T M$ & $d P / d T$ & $d^{2} P / d T^{2}$ & & \\
\hline 54.353 & 0.0015 & 0.00046 & 0.00012 & 0.0015 & 0.00046 & 0.00012 & -0.197 & -0.514 \\
\hline 60.000 & .0072 & .00182 & .00040 & .0072 & .00181 & .00039 & -.448 & -.478 \\
\hline 65.000 & .0230 & .00491 & .00089 & .0229 & .00489 & .00088 & -.373 & -.206 \\
\hline 70.000 & .0616 & .01120 & .00169 & .0614 & .01118 & .00169 & -.198 & .042 \\
\hline 75.000 & .1433 & .02241 & .00285 & .1430 & .02240 & .00286 & -.038 & .168 \\
\hline 80.000 & .2970 & .04034 & .00438 & .2967 & .04037 & .00439 & .058 & .168 \\
\hline 85.000 & .5608 & .06674 & .00623 & .5608 & .06680 & .00624 & .085 & .084 \\
\hline 90.180 & .9997 & .10464 & .00845 & 1.0000 & .10470 & .00844 & .062 & -.035 \\
\hline 95.000 & 1.6108 & .15074 & .01072 & 1.6113 & .15077 & .01070 & .017 & -.125 \\
\hline 100.000 & 2.5088 & .21056 & .01323 & 2.5092 & .21049 & .01321 & -.030 & -.160 \\
\hline 105.000 & 3.7379 & .28328 & .01587 & 3.7377 & .28311 & .01586 & -.059 & -.112 \\
\hline 110.000 & 5.3640 & .36946 & .01861 & 5.3629 & .36925 & .01862 & -.057 & .013 \\
\hline 115.000 & 7.4557 & .46958 & .02145 & 7.4537 & .46946 & .02148 & -.025 & .172 \\
\hline 120.000 & 10.0838 & .58410 & .02438 & 10.0818 & .58425 & .02445 & .026 & .281 \\
\hline 125.000 & 13.3217 & .71364 & .02746 & 13.3214 & .71415 & .02753 & .072 & .239 \\
\hline 130.000 & 17.2466 & .85906 & .03075 & 17.2494 & .85976 & .03075 & .081 & -.017 \\
\hline 135.000 & 21.9410 & 1.02171 & .03438 & 21.9467 & 1.02206 & .03424 & .034 & -.395 \\
\hline 140.000 & 27.4959 & 1.20376 & .03857 & 27.5013 & 1.20319 & .03836 & -.048 & -.529 \\
\hline 145.000 & 34.0172 & 1.40910 & .04382 & 34.0178 & 1.40801 & .04391 & -.077 & .207 \\
\hline 150.000 & 41.6391 & 1.64619 & .05178 & 41.6383 & 1.64736 & .05255 & .071 & 1.487 \\
\hline 151.000 & 43.3115 & 1.69914 & .05421 & 43.3123 & 1.70106 & .05489 & .113 & 1.255 \\
\hline 152.000 & 45.0383 & 1.75483 & .05734 & 45.0413 & 1.75723 & .05751 & .137 & 0.294 \\
\hline 153.000 & 46.8224 & 1.81427 & .06189 & 46.8277 & 1.81617 & .06044 & .105 & -2.346 \\
\hline 154.000 & 48.6688 & 1.87999 & .07097 & 48.6746 & 1.87824 & .06374 & -.093 & -10.180 \\
\hline 154.100 & 48.8572 & 1.88716 & .07263 & 48.8628 & 1.88463 & .06410 & -.134 & -11.744 \\
\hline 154.200 & 49.0463 & 1.89452 & .07462 & 49.0516 & 1.89105 & .06445 & -.183 & -13.625 \\
\hline 154.300 & 49.2361 & 1.90210 & .07711 & 49.2410 & 1.89752 & .06481 & -.241 & -15.944 \\
\hline 154.400 & 49.4267 & 1.90997 & .08038 & 49.4311 & 1.90402 & .06518 & -.312 & -18.911 \\
\hline 154.500 & 49.6181 & 1.91822 & .08505 & 49.6218 & 1.91055 & .06555 & -.400 & -22.927 \\
\hline 154.600 & 49.8104 & 1.92708 & .09278 & 49.8132 & 1.91713 & .06592 & -.516 & -28.950 \\
\hline 154.700 & 50.0036 & 1.93710 & .11141 & 50.0052 & 1.92374 & .06630 & -.690 & -40.487 \\
\hline
\end{tabular}

TABLE 4. Comparisons of $\mathrm{d}^{2} \mathrm{P} / \mathrm{dT}^{2}$ for oxygen

\begin{tabular}{|c|c|c|}
\hline \multicolumn{3}{|c|}{$\bar{C}_{v} / T=-d^{2} G / d T^{2}+\left(d^{2} P / d T^{2}\right) \cdot v$} \\
\hline \multirow[b]{2}{*}{$\mathrm{T}, \mathrm{K}$} & \multicolumn{2}{|c|}{$d^{2} P / d T^{2}$, atm $/ K^{2}$} \\
\hline & V.P. eq (3a) & $\operatorname{Expt}{ }^{\prime} l \bar{C}_{v}$ \\
\hline 60.00 & 0.0004 & $0.0007 \pm 100 \%$ \\
\hline 65.00 & .0009 & .0012 \\
\hline 70.00 & .0017 & .0020 \\
\hline 75.00 & .0029 & .0031 \\
\hline 80.00 & .0044 & .0046 \\
\hline 85.00 & .0062 & .0064 \\
\hline 90.18 & .0085 & .0084 \\
\hline 95.09 & .0107 & .0106 \\
\hline 100.00 & .0132 & .0130 \\
\hline 105.00 & .0159 & .0155 \\
\hline 110.00 & .0186 & .0182 \\
\hline 115.00 & .0215 & .0211 \\
\hline 120.00 & .0244 & .0240 \\
\hline 125.00 & .0275 & $.0271 \pm 2 \%$ \\
\hline
\end{tabular}


TABLE 5. Constants for (3) with logarithmic $\mathrm{F}(\mathrm{x})$

\begin{tabular}{|c|c|c|c|}
\hline & Nitrogen & Oxygen & Fluorine \\
\hline A......... & -0.0218 & -0.2208 & -0.1109 \\
\hline B.... & 3.1969 & 1.2267 & 1.2507 \\
\hline C... & 0.1099 & 0.3096 & 0.1913 \\
\hline
\end{tabular}

The oxygen data used here may have a uniquely high precision near the critical point. As (3a) gives an excellent representation of these data, this is good evidence that the vapor pressure formula indeed should be nonanalytic at the critical point, as was suggested by Yang and Yang in 1964.

\section{Comments}

The following comments have been received, of interest in the rapidly developing theory of critical states. Griffiths and Rushbrooke have proven that if $d^{2} P / d T^{2}$ diverges as $\left|T-T_{c}\right|^{-\theta}$, then $\theta<\alpha^{\prime}+\beta[3 \mathrm{~b}]$, which brings up the lower limit on $\epsilon$ found in the present report for oxygen vapor pressure data.

The scaling laws limit the value of $\epsilon$ even further, namely to close to 2 . These laws are postulates, not proofs [12]. Kecently, a nonanalytic scaling law vapor pressure equation has been used for $\mathrm{CO}_{2}$ near the critical point [13].

\section{References}

[1] Miller, D. G., Estimating vapor pressures-a comparison of equations, Ind. Eng. Chem. 56 (3), 46-57 (1964).

[2] Yang, C. N., and Yang, C. P., Critical point in liquid-gas transitions, Phys. Rev. Letters, 13 (9), 303-5 (1964).

[3a] Fisher, M. E., in Critical Phenomena, NBS Miscellaneous Publication 273, M. S. Green and J. V. Sengers, Editors, (U.S. Government Printing Office, Washington, D.C. 20402, 1966).

[3b] Temperley, H. N. V., Rowlinson, J. S., and Rushbrooke, G. S., editors, Physics of Simple Liquids (North-Holland Publishing Co., Amsterdam, 1968).

[4] Straty, G. C., and Prydz, Rolf, The vapor pressure of liquid fluorine, presented at the Cryogenic Engineering Conference, University of California, Los Angeles, June 17, 1969.

[5] Hoge, H. J., Vapor pressure and fixed points of oxygen, and heat capacity in the critical region, J. Res. NBS 44, 321-345 (1950), RP2081.

[5a] Muijlwijk, R., Moussa, M. R., and van Dijk, H., The vapour pressure of liquid oxygen, Physica 32 (5), 805-22 (1966).

[6] Strobridge, T. R., The thermodynamic properties of nitrogen from 64 to $300 \mathrm{~K}$ between 0.1 and 200 atmospheres, NBS Tech. Note 129 (Jan. 1962).

[7] Armstrong, G. T., The vapor pressure of liquid nitrogen, J. Res. NBS 53, 263-66 (1954), RP2543.

[8] Friedman, A. S., and White, D., The vapor pressure of liquid nitrogen, J. Am. Chem. Soc. 72 (9), 3931-32 (1950).

[9] Hust, J. G., and Stewart, R. B., Private communication, this laboratory, Feb. 16, 1965. Reported by R. B. Stewart, The thermodynamic properties of oxygen, Dissertation, Dept. Mech. Engr'g., Univ. of Iowa, June 1966.

[10] Moussa, M. R., Muijlwijk, R., and van Dijk, H., The vapour pressure of liquid nitrogen, Physica 32, 900-12 (1966).

[11] Goodwin, R. D., and Weber, L. A., Specific heats of oxygen at coexistence, J. Res. Nat. Bur. Stand. (U.S.) 73A, No. 1, 15-24 (Feb. 1969).

[12] Vicentini-Missoni, M., Sengers, J. M. H. Levelt, and Green, M. S., Thermodynamic anomalies of $\mathrm{CO}_{2}, \mathrm{Xe}$, and $\mathrm{He}^{4}$ in the critical region, Phys. Rev. Letters 22, No. 9, 389-393 (1969).

[13] Sengers, J. M. H. Levelt, Chen, W. T., and Vicentini-Missoni, M., Vapor pressure of $\mathrm{CO}_{2}$ near the critical point, Bulletin of the Am. Phys. Soc., Series II, 14, No. 4, 593 (April 1969).

(Paper 73A5-566) 